Vinflunine is a novel microtubule inhibitor with high antitumor activity against transitional cell carcinoma of the urothelial tract (TCCU), a disease which is associated with a very poor prognosis. There is currently no standard second-line treatment for patients with advanced TCCU after failure of platinum therapy although vinflunine has shown promising activity in phase II trials. These results led Bellmunt and colleagues to carry out a randomized multinational phase III trial of vinflunine as second-line treatment for advanced TCCU.

The authors compared best supportive care plus vinflunine with best supportive care alone in patients who had disease progression after first-line treatment with a platinum-containing agent. Overall survival was the main outcome measure. A total of 370 patients were randomly assigned $2: 1$ to the study $(n=253)$ or control arm $(n=117)$.

Neutropenia, febrile neutropenia, anemia, fatigue and constipation were the main grade 3 or 4 toxic effects in the study group. Multivariate analysis revealed that vinflunine had a statistically significant effect on overall survival $(P=0.036)$ and reduced the risk of death by $23 \%$. The median overall survival, overall response rate, disease control and progression-free survival were all improved in the best supportive care plus vinflunine group. The objective of a median 2-month survival advantage with best supportive care plus vinflunine was met in the intent-to-treat population.

The results of this study show that vinflunine provides a survival advantage for the second-line treatment of TCCU, and has an acceptable safety profile. It is, therefore, a reasonable treatment option for patients with TCCU who have experienced disease progression after first-line platinum therapy.

\title{
Mandy Aujla
}

Original article Bellmunt, J. et al. Phase III trial of vinflunine plus best supportive care compared with best supportive care alone after a platinum-containing regimen in patients with advanced transitional cell carcinoma of the urothelial tract. J. Clin. Oncol. 27, 4454-4461 (2009). 